\title{
The A-factor Regulatory Cascade in the Regulation of Physiological and Morphological Development in Streptomyses griseus
}

\author{
Yasuo Ohnishi* \\ Department of Biotechnology, Graduate School of Agriculture and Life Sciences, \\ The University of Tokyo, Bunkyo-ku, Tokyo 113-8657, Japan \\ (Received Mar. 10, 2003)
}

\section{INTRODUCTION}

The genus Streptomyces comprises Gram-positive, soilinhabiting, filamentous bacteria. The species within this genus embody complex morphological differentiation resembling that of filamentous fungi, making this a model genus of prokaryotes for the study of multicellular differentiation. Another characteristic of this genus is the ability of its member species to produce a wide variety of secondary metabolites, including antibiotics and biologically active substances, making Streptomyces important industrial microorganisms. Secondary metabolite formation is sometimes called physiological differentiation, because it occurs during the idiophase after the main period of rapid vegetative growth and assimilative metabolism. In a streptomycin producer, Streptomyces griseus, a low-molecularweight chemical signaling molecule, A-factor (2-isocapryloyl-3R-hydroxymethyl- $\gamma$-butyrolactone), triggers both morphological and physiological differentiation. A-factor was originally discovered by Khokhlov et al. in 1967 as a diffusible extracellular molecule that causes sporulation of a bald mutant of $S$. griseus $^{1,2)}$. Khokhlov et al. also found that A-factor is essential not only for aerial mycelium formation, but for streptomycin biosynthesis in this mutant. Hara and Beppu rediscovered A-factor in 1982 and confirmed the observations by Khokhlov et al. with stereochemically synthesized A-factor at concentrations as low as $10^{-9} \mathrm{M}^{3}$. Beppu, Horinouchi, and their colleagues have analyzed the regulatory mechanism triggered by Afactor using recombinant DNA techniques ${ }^{4-6)}$. Since 1996, I have investigated the regulation of morphological and physiological differentiation in Streptomyces in Prof. Horinouchi's laboratory. This article summarizes the progress of our studies on the A-factor regulatory cascade.

\section{Identification of $a d p A$ as a target gene of the A-factor receptor $^{7)}$}

In S. griseus, the presence of A-factor at extremely low concentrations triggers streptomycin production and aerial mycelium formation by binding a repressor-type receptor protein $(\mathrm{ArpA})$ and dissociating it from the $\mathrm{DNA}^{8,9)}$.
Although in vitro experiments established a consensus sequence (a 22 bp palindrome, one half of which is 5'GG(T/C)CGGT(A/T)(T/C) G(T/G)-3') to which ArpA binds ${ }^{9)}$, the target genes of ArpA in vivo had been unknown. Identification of the target genes of ArpA was a crucial task in elucidating the A-factor regulatory cascade.

In the streptomycin biosynthetic gene cluster in $S$. griseus, strR encodes a pathway-specific transcriptional activator required to transcribe other genes in the cluster ${ }^{10 \text {, }}$ 11). An A-factor-dependent protein (AdpA) had been identified to bind the region upstream of the $s t r R$ promoter and activate its transcription ${ }^{12,13)}$. Therefore, AdpA was regarded as an important regulator that transmitted the upstream A-factor signal directly to the streptomycin production genes via $s t r R$. Starting with a crude lysate prepared from mycelium of the $S$. griseus wild-type strain, we purified AdpA to near homogeneity. The amino terminal and internal amino acid sequences of AdpA were determined and a part of the $a d p A$ sequence was cloned by PCR. Then a DNA fragment containing whole $a d p A$ sequence was cloned by a colony hybridization method, using the partial $a d p A$ sequence as a probe. $a d p A$ was found to encode a 405-amino-acid protein that contained a helix-turn-helix (HTH) DNA-binding motif in the central portion, as determined by nucleotide sequencing of the cloned fragment. Recombinant AdpA produced in Escherichia coli bound and recognized the upstream activation sequence for strR. Consistent with the idea that AdpA serves as a transcriptional activator for $s t r R$, AdpA shows significant homology to some positive transcriptional regulators in the AraC/XylS family, especially around the HTH motif.

High-resolution S1 mapping determined the transcriptional start point of $a d p A$ to be the C $263 \mathrm{bp}$ upstream from the initiation codon. The -35 and -10 regions of $a d p A$ were found to overlap with a sequence highly similar to the consensus sequence of the ArpA-binding site. As expected, ArpA bound the promoter region of $a d p A$ in the absence of A-factor, but did not do so in the presence of A-factor. Furthermore, exogenous addition of A-factor to the ArpADNA complex immediately dissociated ArpA from the DNA. Consistent with this finding, S1 nuclease mapping

*Corresponding author. Phone: +81-3-5841-5126. Fax: +81-3-5841-5337. E-mail: ayasuo@ mail.ecc.u-tokyo.ac.jp 
showed that $a d p A$ was transcribed only in the presence of A-factor. Thus, the promoter of $a d p A$ proved to be a target of ArpA.

\section{AdpA as a positive regulator for streptomycin biosynthesis $^{7)}$}

For determination of the in vivo function of $a d p A$ in $S$. griseus, an adpA-disrupted strain was constructed. The $a d p A$ disruption had no effect on growth in liquid or on a solid medium. As expected, in the $\triangle a d p A$ strain, no transcription of $s t r R$ was detected and no streptomycin was produced. Furthermore, overexpression of $a d p A$ caused the wild-type $S$ griseus strain to produce large amounts of streptomycin at an earlier growth stage. All this data suggested that AdpA is a transcriptional activator for $s t r R$, a pathway-specific transcriptional gene in a streptomycin biosynthetic gene cluster.

\section{Signal relay from A-factor to the streptomycin biosynthetic genes ${ }^{7)}$}

As described above, the signal relay from A-factor to the streptomycin biosynthetic genes was revealed to be Afactor to ArpA to AdpA to StrR to the streptomycin biosynthetic genes (Fig. 1). A-factor is likely produced by

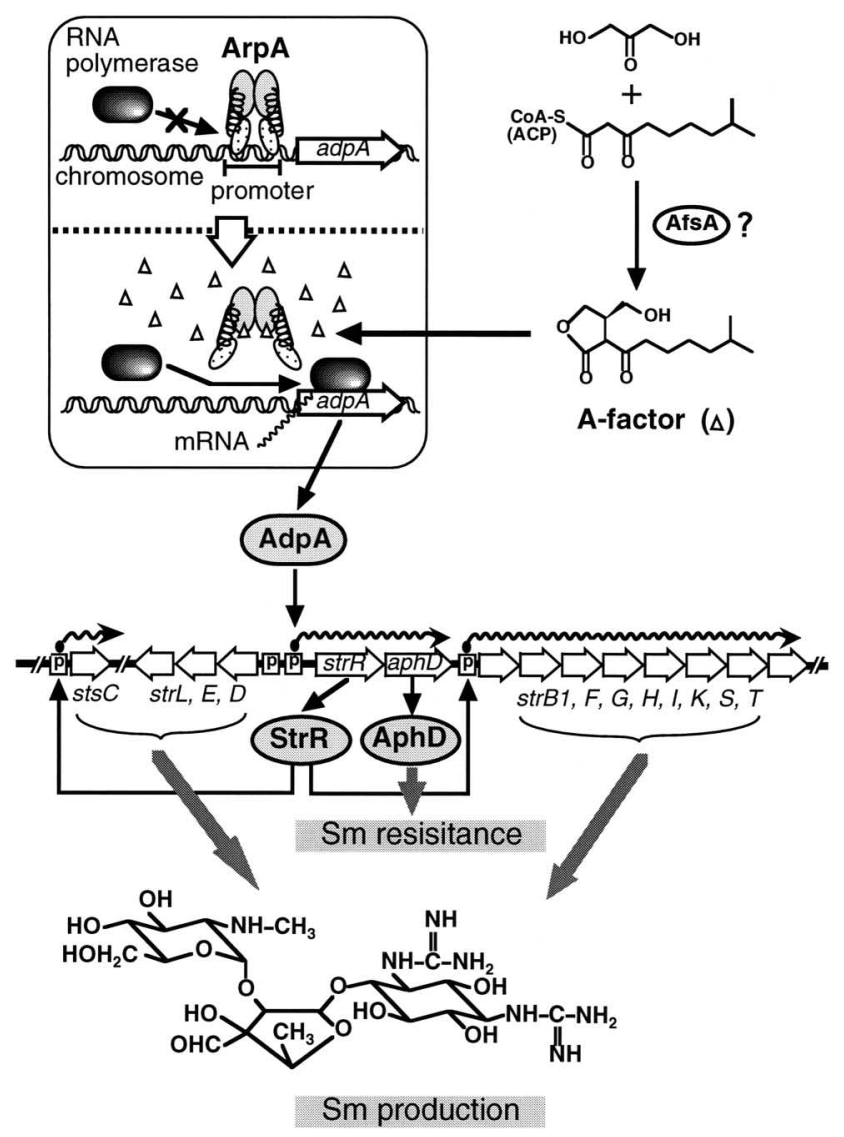

Fig. 1. How streptomycin production and resistance are triggered by A-factor in S. griseus.

Sm; streptomycin. See text (chapter 3) for details. the action of AfsA, gradually accumulating in a growthdependent manner. When the concentration of A-factor reaches a certain critical level, it binds ArpA that has bound and repressed the promoter of $a d p A$ in an early growth stage and dissociates ArpA from the promoter, causing transcription of $\operatorname{adpA}$. The AdpA thus induced binds the upstream activation sequence of $s t r R$ and activates its transcription. The pathway-specific transcriptional activator StrR then induces transcription of most of the streptomycin biosynthetic genes by binding multiple sites in the gene cluster ${ }^{12,13)}$, leading to biosynthesis of streptomycin. The timing of the onset of streptomycin biosynthesis is therefore determined by the intracellular concentration of A-factor. aphD, encoding a major streptomycin resistance determinant, strepyomycin-6-phosphotransferase, is under the control of the $a d p A$-dependent promoter, because $a p h D$ is transcribed mainly by readthrough from the $s t r R$ promoter ${ }^{10)}$. Direct dependence of the $a p h D$ transcription on the A-factor-inducible strR promoter accounts for the prompt induction of streptomycin resistance and would help achieve a rapid increase in self-resistance to avoid suicide just before induction of streptomycin biosynthesis.

\section{AdpA regulon: multiple targets of $\operatorname{AdpA}$}

As described above, AdpA was originally identified as a transcriptional activator for strR. However, characterization of the $\triangle a d p A$ strain indicated a pleiotropic function of $a d p A$. The $\triangle a d p A$ strain failed to produce not only streptomycin, but a diffusible yellow pigment, production of which is also triggered by A-factor ${ }^{14)}$. Furthermore, the $\triangle a d p A$ strain failed to make aerial mycelium; it grew as a substrate mycelium for a prolonged incubation period ${ }^{7)}$. These observations implied that AdpA controls multiple, unlinked genes necessary for morphological and physiological differentiation, and prompted us to isolate the multiple genes as DNA fragments bound by AdpA.

For this purpose, a library of fragmented chromosomal DNA of about 300 to 500 bp from S. griseus was constructed by HaeIII digestion, followed by attachment of a linker at the ends. The library was incubated with AdpA to allow formation of protein-DNA complexes. The complex was separated from free DNA by a gel mobility shift, and the DNA in the complex was purified by extraction from the gel pieces. The purified DNA was then amplified by PCR with the linkers as primers. For enrichment of the DNA fragments, the PCR product was subjected to second and further cycles of the gel mobility shift-PCR procedure. Repeated experiments yielded 60 different DNA fragments specifically bound by $\operatorname{AdpA}^{14,15)}$. Although it is unlikely that all of these AdpA-binding sites have functional roles in vivo, we were able to isolate several novel target genes of AdpA by this method, as described below.

\section{An extracytoplasmic function sigma factor $\operatorname{Ads} \mathbf{A}^{14)}$}

The DNA fragment most abundantly obtained in the gel 
mobility shift-PCR procedure was located in front of a gene, named $a d s A$, encoding an extracytoplasmic function (ECF) $\sigma$ factor belonging to a subgroup of the primary $\sigma^{70}$ family. As expected, transcription of adsA depended on Afactor and adpA. AdpA bound one site, position about +25 , just downstream of the transcriptional start point of $a d s A$. The unusual position of the binding site of AdpA as an activator suggests the presence of a mechanism by which AdpA activates transcription of $a d s A$ in some unknown way. Our preliminary experiments showed that AdpA was required for a stable open complex formation of the $a d s A$ promoter by RNA polymerase (from our unpublished results). The in vivo function of adsA was determined to be involved in aerial mycelium formation and not in secondary metabolite production, because an $a d s A$-disrupted strain failed to form aerial mycelium but still produced streptomycin and a yellow pigment. bldN, the orthologue of adsA in Streptomyces coelicolor A3(2), is also required for aerial mycelium formation ${ }^{16}$. A gene (or genes) that is transcribed by RNA polymerase containing $\sigma^{\text {AdsA }}$ remains to be identified, while $\sigma^{\text {BldN }}$ is required for the transcription from P1 promoter of bldM in S. coelicolor A3(2) ${ }^{16)}$.

\section{Extracellular metalloendopeptidase SgmA (SGMP II) ${ }^{17)}$}

A region upstream of a zinc-containing metalloendpeptidase gene, named $\operatorname{sgmA}$, was found among DNA fragments isolated in the gel mobility shift-PCR procedure. The amino acid sequence from Gly-234 to Gly-567 of SgmA was found to match completely that of the metalloendopeptidase (SGMP II) purified from S. griseus K$1^{18)}$, revealing that $\operatorname{SgmA}$ was primarily translated in a prepro form (Met-1 to Phe-681), with a large pre-pro region in its $\mathrm{NH}_{2}$-terminal portion (Met-1 to Asn-233) and a large pro region in its COOH-terminal region (Lys-568 to Phe681). Transcription of $\operatorname{sgmA}$ was completely abolished in the $\triangle a d p A$ strain, indicating its dependence on $a d p A$ and A-factor. AdpA bound two sites in the region upstream of the $\operatorname{sgmA}$ promoter; one at about nucleotide position -60 (A site), and the other about position -260 (B site). Transcriptional analysis with mutated promoters showed that the A site was essential for switching on sgmA transcription and that the B site was necessary for marked enhancement of the transcription in a late-stage growth phase. Disruption of the chromosomal sgmA gene delayed aerial mycelium formation by half a day. No differences were observed in growth and in secondary metabolite production between the wild-type and the sgmA-disrupted strains. It is therefore suggested that $\mathrm{SgmA}$ is associated with the programmed morphological development of $S$. griseus. It is noteworthy that several extracellular serine protease genes, such as $s p r A, \operatorname{spr} B, \operatorname{spr} D$, and $s p r T$, were also found to be under the control of $a d p A$ and A-factor (from our unpublished results). It is presumed that, in consideration of the emergence of aerial hyphae into the air from substrate hyphae, these proteases hydrolyze proteins assimilated into the substrate hyphae for reuse of amino acids for aerial mycelium formation.

\section{SsgA required for septation in aerial hyphae ${ }^{19)}$}

We also identified a DNA fragment located in front of $s s g A$, which had been reported to be essential for septum formation in aerial mycelium in S. griseus $^{20)}$ and $S$. coelicolor A3(2) ${ }^{21}$. ssgA had two transcriptional start points, one starting at the 124 nucleotide (p1) and the other starting at the 79 nucleotide (p2) upstream of the start codon of $s s g A$. Transcription of $s s g A$ from both $\mathrm{p} 1$ and $\mathrm{p} 2$ depended on $\operatorname{adpA}$ and A-factor. AdpA bound three sites at nucleotide positions about -235 (site 1), -110 (site 2), and +60 (site 3) with respect to the transcriptional start point, $\mathrm{p} 1$, of $s s g$. Transcriptional analysis with mutated promoters indicated that of the three binding sites, only sites 1 and 2 were required for transcriptional activation of $s s g A$. An $s s g A$-disrupted strain formed aerial hyphae, but did not form spores, indicating that $s s g A$ is a member of the whi genes. This is an exceptional example, showing that A-factor controls a step for morphological development after aerial hyphae have formed; all steps controlled by A-factor hitherto observed have been involved in transitions from substrate hyphae to aerial hyphae.

The presence of many genes, all of which are simultaneously activated by AdpA at a specific point in the growth phase, means that the signal from A-factor is greatly amplified at this regulatory step via AdpA as an amplifier (Fig. 2). In the A-factor regulatory pathway, afs $A$, arpA, and $a d p A$ are common to secondary metabolism and morphological differentiation, because the disruption of either of these genes simultaneously affects both phenotypes. AdpA therefore serves as a switch for many genes, each of which is necessary for respective phenotypes under the control of A-factor.

\section{Concluding remarks}

The results of this study come close to providing an overall picture of the A-factor regulatory cascade. It is safe to say that the A-factor regulatory cascade is the most important regulatory system in the regulation of morphological and physiological differentiation in S. griseus, although some other regulatory systems are also found in the strain. Many Streptomyces species produce $\gamma$-butyrolactones, most of which have been shown to act as signals for the onset of antibiotic production. A-factor is exceptionally required not only for antibiotic production, but for morphological differentiation in S. griseus. Streptomyces species presumably developed $\gamma$-butyroloctones during the course of their evolution as 'hormonal regulators' to control different stages of physiological and/or morphological differentiation in the regulatory hierarchy. Due to their filamentous growth, Streptomyces may have developed diffusible $\gamma$-butyrolactone regulatory systems to facilitate communication between cells in an individual mycelium. 


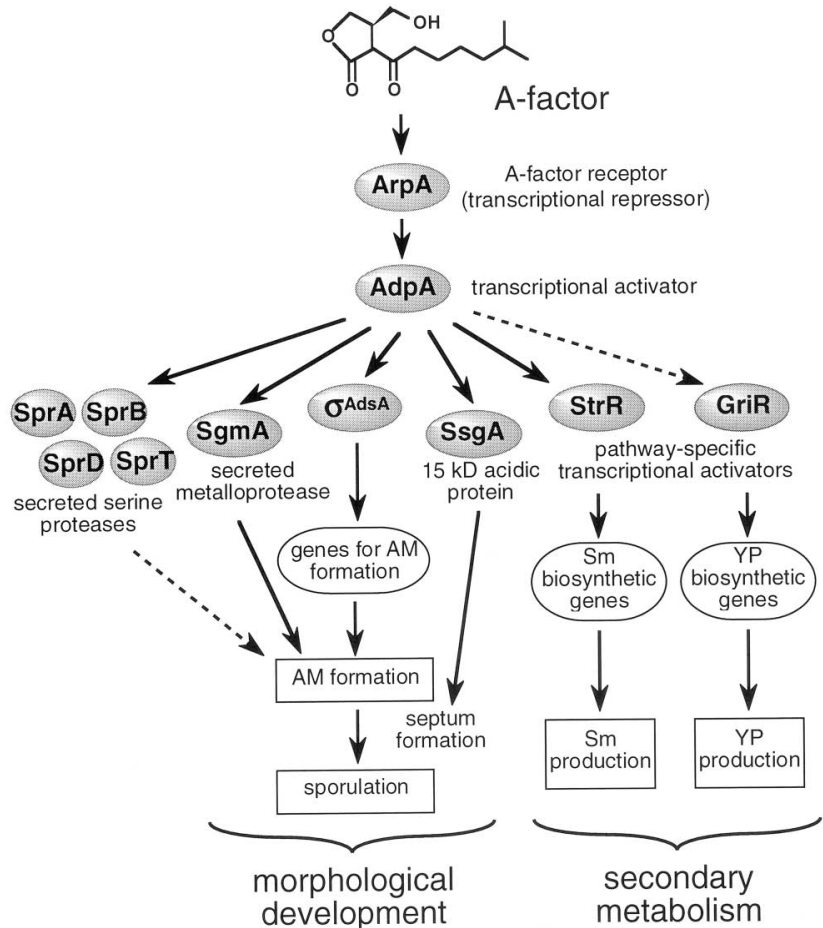

Fig. 2. The A-factor regulatory cascade leading to physiological and morphological development in S. griseus.

AM; aerial mycelium, Sm; streptomycin, YP; an A-factor-dependent yellow pigment. We have cloned the yellow pigment biosynthetic gene cluster and identified the pathway-specific transcriptional activator gene, griR, in the cluster (from our unpublished results). See text (chapter 4) for details.

The filamentous mycelium of Streptomyces has many branches and forms a complex network, in which some branches are close enough to easily communicate with each other by diffusible low-molecular-weight chemicals. The signaling system between physically separate individual cells in the same mycelium can be regarded as hormonal regulation, rather than the quorum sensing system found in Gram-negative single-cell bacteria. In a complex filamentous mycelium network of Streptomyces, $\gamma$ butyroloctone autoregulators are regarded as important for synchronizing the physiological and/or morphological differentiation of individual cells in the same mycelium. However, because the $\gamma$-butyroloctone autoregulator produced by a mycelium is accepted by other neighboring hyphae, it may also play a crucial role in cell-to-cell communications between individual hyphae, similar to the quorum-sensing system. Presumably, this system would prove advantageous for survival within the ecosystem.

\section{ACKNOWLEDGEMENTS}

It was my great honor to receive the Hamada Award of the Society for Actinomycetes Japan (SAJ) in 2002. I am grateful to Prof. Haruo Ikeda for his recommendation and to the members of the award nomination committee. I would like to express my gratitude and appreciation to Prof. Sueharu Horinouchi for his kind and careful direction. These studies were done in fine cooperation with students in the Laboratory of Fermentation and Microbiology. I am deeply indebted to all of these colleagues.

\section{REFERENCES}

1) Khokhlov, A. S.; I. I. Tovarova, L. N. Borisova, S. A. Pliner, L. A. Shevchenko, E. Y. Kornitskaya, N. S. Ivkina, \& I. A. Rapoport: The A-factor, responsible for streptomycin biosynthesis by mutant strains of Actinomyces streptomycini. Dokl. Akad. Nauk. SSSR 177: 232-235, 1967

2) Khokhlov, A. S.: Results and perspectives of actinomycete autoregulators studies. In Biology of Actinomycetes '88. Ed., Y. Okami, T. Beppu \& H. Ogawara, pp. 338-345, Japan Scientific Societies Press, Tokyo, 1988

3) Hara, O. \& T. Beppu: Mutants blocked in streptomycin production in Streptomyces griseus - the role of A-factor. J. Antibiot. 35: 349-358, 1982

4) Horinouchi, S. \& T. Beppu: Autoregulatory factors and communication in actinomycetes. Annu. Rev. Microbiol. 46: 377-398, 1992

5) Horinouchi, S. \& T. Beppu: A-factor as a microbial hormone that controls cellular differentiation and secondary metabolism in Streptomyces griseus. Mol. Microbiol. 12: 859-864, 1994

6) Horinouchi, S.: A microbial hormone, A-factor, as a master switch for morphological differentiation and secondary metabolism in Streptomyces griseus. Front. Biosci. 7: d2045-2057, 2002

7) Ohnishi, Y.; S. Kameyama, H. Onaka \& S. Horinouchi: The A-factor regulatory cascade leading to streptomycin biosynthesis in Streptomyces griseus: identification of a target gene of the A-factor receptor. Mol. Microbiol. 34: 102-111, 1999

8) Onaka, H.; N. Ando, T. Nihira, Y. Yamada, T. Beppu \& S. Horinouchi: Cloning and characterization of the A-factor receptor gene from Streptomyces griseus. J. Bacteriol. 177: 6083-6092, 1995

9) Onaka, H. \& S. Horinouchi: DNA-binding activity of the Afactor receptor protein and its recognition DNA sequences. Mol. Microbiol. 24: 991-1000, 1997.

10) Vujaklija, D.; K. Ueda, S.-K. Hong, T. Beppu \& S. Horinouchi: Identification of an A-factor-dependent promoter in the streptomycin biosynthetic gene cluster of Streptomyces griseus. Mol. Gen. Genet. 229: 119-128, 1991

11) Vujaklija, D.; S. Horinouchi \& T. Beppu: Detection of an Afactor-responsive protein that binds to the upstream activation sequence of strR, a regulatory gene for streptomycin biosynthesis in Streptomyces griseus. J. Bacteriol. 175: 2652-2661, 1993

12) Distler, J.; K. Mansouri, G. Mayer, M. Stockmann, \& W. Piepersberg: Streptomycin biosynthesis and its regulation in streptomycetes. Gene 115: 105-111, 1992

13) Retzlaff, L. \& J. Distler: The regulator of streptomycin gene expression, StrR, of Streptomyces griseus is a DNA binding activator protein with multiple recognition sites. Mol. Microbiol. 18: 151-162, 1995 
14) Yamazaki, H.; Y. Ohnishi \& S. Horinouchi: An A-factordependent extracytoplasmic function sigma factor $\left(\sigma^{\text {AdsA }}\right)$ that is essential for morphological development in Streptomyces griseus. J. Bacteriol. 182: 4596-4605, 2000

15) Horinouchi, S.; H. Onaka, H. Yamazaki, S. Kameyama \& Y. Ohnishi: Isolation of DNA fragments bound by transcriptional factors, AdpA and ArpA, in the A-factor regulatory cascade. Actinomycetologica 14: 37-42, 2000

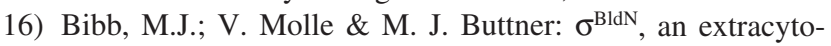
plasmic function RNA polymerase sigma factor required for aerial mycelium formation in Streptomyces coelicolor A3(2). J. Bacteriol. 182: 4606-4616, 2000

17) Kato, J.; A. Suzuki, H. Yamazaki, Y. Ohnishi \& S. Horinouchi: Control by A-factor of a metalloendopeptidase gene involved in aerial mycelium formation in Streptomyces griseus. J. Bacteriol. 184: 6016-6025, 2002
18) Kumazaki, T.; K. Kajiwara, S. Kojima, K. Miura \& S. Ishii: Interaction of Streptomyces subtilisin inhibitor (SSI) with Streptomyces griseus metallo-endopeptidase II (SGMP II). J. Biochem. (Tokyo) 114: 570-575, 1993

19) Yamazaki, H.; Y. Ohnishi \& S. Horinouchi: Transcriptional switch on of ssgA by A-factor, which is essential for spore septum formation in Streptomyces griseus. J. Bacteriol. 185: 1273-1283, 2003

20) Jiang, H. \& K. E. Kendrick: Characterization of $s s f R$ and ss $g A$, two genes involved in sporulation of Streptomyces griseus. J. Bacteriol. 182: 5521-5529, 2000

21) van Wezel, G. P.; J. van der Meulen, S. Kawamoto, R. G. Luiten, H. K. Koerten \& B. Kraal: $\operatorname{ssg} A$ is essential for sporulation of Streptomyces coelicolor A3(2) and affects hyphal development by stimulating septum formation. J. Bacteriol. 182: 5653-5662, 2000 